\title{
Area Under the Curve Ratio Infinity Predicted
}

National Cancer Institute

\section{Source}

National Cancer Institute. Area Under the Curve Ratio Infinity Predicted. NCI Thesaurus.

Code C156577.

The ratio of two AUC infinity predicted values. 